\title{
Löst die Gemeinschaftspraxis die klassische Einzelpraxis ab?
}

\author{
Die medizinische Grundversorgung steht vor einem tiefgreifenden Strukturwandel, \\ der bereits begonnen hat. Gemeinschaftspraxen boomen, angetrieben von demogra- \\ fischen Entwicklungen, fortschreitender Digitalisierung und wachsender Komplexität \\ im Gesundheitswesen.
}

\section{Lea Maurer}

Verantwortliche Marketing und Kommunikation Exhibit \& More AG
Korrespondenz:

Lea Maurer

Exhibit \& More AG

Bruggacherstrasse 26

Postfach 185

CH-8117 Fällanden

Tel. 0448063333 (Zentrale)

press[at]exhibit.ch
Es sind lange Arbeitstage, die der «gute alte Hausarzt» täglich zu bewältigen hat. Als - mehrheitlich männlicher - «Einzelkämpfer» mit eigener Praxis, kostspieligen medizinischen Geräten und zu betreuenden Angestellten hat er eine hohe finanzielle Verantwortung zu tragen und muss sich neben seiner medizinischen Tätigkeit um die unternehmerischen sowie administrativen Belange seines KMU kümmern. In den nächsten zehn Jahren werden knapp 3700 dieser «guten alten Hausärzte» pensioniert [1]. Schon jetzt sind 48 Prozent der Hausärzte über 55 Jahre alt [1].

\section{Lieber angestellt und weniger Risiko tragen} Gemäss dem Innerschweizer Praxisvermittler Roland Willi haben Inhaber von Einzelpraxen bereits heute ein akutes Problem bei der Nachfolgeregelung:

\section{Besonders Frauen wollen nicht mit ihrem Job «verheiratet sein»}

Mit der Feminisierung des Berufsstands zusammenhängend, lässt sich neben dem Trend zum Angestelltenverhältnis ein zweiter Trend identifizieren: Der Wunsch nach Teilzeitarbeit. Gemäss FMH-Ärztestatistik arbeiten im ambulanten Bereich 31 Prozent der Männer Teilzeit, während es bei den Frauen mit 73 Prozent mehr als doppelt so viele sind [3].

Auch Roland Willi stellt fest, dass junge Ärztinnen und Ärzte heute lieber Teilzeit arbeiten. Peter Sauter, Geschäftsführer von «eastcare», einem ökonomisch-medizinischen Praxis-Dienstleister, spricht von einem deutlichen Trend zur Teilzeitarbeit: «Der gesellschaftliche Trend zu neuen Familienmodellen macht auch vor Hausärzten nicht halt. Die nachrückende Generation möchte nicht mehr «verheira-

\section{«Der gesellschaftliche Trend zu neuen Familienmodellen macht auch vor Hausärzten nicht halt.»}

«Generell sind junge Ärztinnen, aber auch Ärzte, heute immer weniger bereit, das finanzielle Risiko als selbständig Erwerbende zu tragen. Gerade Frauen zeigen hier eine klare Präferenz zum Angestelltenverhältnis.» War es früher die absolute Regel, als selbständiger Hausarzt zu praktizieren, erwägen heute immerhin schon 41 Prozent der angehenden Allgemeinmediziner, auf Angestelltenbasis mit fixem Salär und geregelten Arbeitszeiten tätig zu sein [2].

In den letzten Jahren ist der Frauenanteil in der Ärzteschaft stetig angestiegen. 2008 lag dieser bei 34,6 Prozent, in der Altersgruppe der 25- bis 29-Jährigen gar bei rund 60 Prozent [3]. Der demografische Wandel zeigt sich auch bei den Studienabschlüssen: Seit 2005 schliessen konstant mehr Frauen als Männer ihr Medizinstudium erfolgreich ab (2009: 61 Prozent) [4]. tet sein» mit ihrem Job. Die demografischen Veränderungen fragen nach neuen Strukturen, um die medizinische Grundversorgung sicherzustellen.»

Gemäss einer Umfrage des Verbands junger Hausärztinnen und -ärzte Schweiz unter ihren Mitgliedern liegt das Wunschpensum bei Frauen zwischen 60 und 70 Prozent, bei Männern um 80 Prozent [2]. Auch wenn die Zahlen nicht repräsentativ sind, vermitteln sie doch ein deutliches Bild der Bedürfnisse und Wünsche der zukünftigen Generation des Berufsstandes.

\section{Mehr Lebensqualität in der Gemeinschafts- praxis?}

Sowohl Peter Sauter wie auch Roland Willi sehen in obengenannten Trends den Wunsch nach mehr Lebensqualität junger Allgemeinmediziner. Gemein- 
schaftspraxen mit ihren deutlich flexibleren Arbeitszeiten und Anstellungsbedingungen berücksichtigen die Bedürfnisse der jungen Berufsgarde in besonderer Weise, etwa wenn es darum geht, Berufstätigkeit und Familie unter einen Hut zu bringen. Silvia Schnidrig, Leiterin Unternehmenskommunikation der «Swica», sieht als Vorteil bestehender Gesundheitszentren zudem, dass Ärzte sich keinen eigenen Kundenstamm aufbauen und die medizinischen Apparaturen nicht selbst anschaffen müssen.

Alle drei Fachleute antworten unisono, dass der klassische «Einzelkämpfer» mehr und mehr ausgedient hat und der Austausch unter Kollegen in Gemeinschaftspraxen einen positiven Effekt auf die Arbeitszufriedenheit hat. Diese Entwicklung bestätigt auch das Stimmungsbild der Befragung von 104 jungen Hausärztinnen und -ärzten in der Schweiz, in der 78 Prozent angegeben haben, sie möchten in einer Gruppenpraxis praktizieren [2].

Gemäss einer Erhebung der FMH-Ärztestatistik hat denn auch der Anteil von Ärztinnen und Ärzten im ambulanten Sektor, die in einer Einzelpraxis arbeiten, seit 2008 um rund 5 Prozent auf 58,6 Prozent abgenommen. Frauen (50,3 Prozent) haben im Vergleich zu Männern (37,1 Prozent) eine höhere Affinität zur (Team-)Arbeit in Gruppenpraxen [1].

\section{Patienten profitieren von Arbeitsteilung}

Auch aus Patientensicht ortet Schnidrig Vorteile von Gemeinschaftspraxen - die über Jahre entstehende Vertrauensbeziehung zwischen Arzt und Patient sei

\section{Steigende Komplexität fordert neue Strukturen -} die IFAS bietet den Überblick

Im Zuge von Managed Care, TARMED und eHealth sind die technologischen Herausforderungen wie auch die Anforderungen an die Zusammenarbeit medizinischer Dienstleister gestiegen. Es braucht zunehmend eine «kritische Praxisgrösse», um die Grundversorgung kosteneffizient zu gewährleisten. Technologische Innovationen bieten die Möglichkeit, Strukturen zu professionalisieren und auf die veränderten Rahmenbedingungen zu reagieren. Die IFAS - Fachmesse für den Gesundheitsmarkt bietet mit ihren rund 370 Ausstellern einen umfassenden Überblick über den «State of the art» der modernen Praxisführung. Mit ihren Foren zu «veränderten Strukturen» sowie zur «Optimierung von Prozessen» im Beschaffungswesen erfahren Messebesucher zudem aus erster Hand, wie sie ihre Praxisdienstleistungen noch effizienter gestalten können.

kommentieren? Nutzen Sie dafür die Kommentarfunktion in der OnlineVersion oder sehen Sie nach, was Ihre Kolleginnen und Kollegen bereits geschrieben haben: www.saez.ch/ aktuelle-ausgabe/ interaktive-beitraege/

\section{Die IFAS vom 21.-24. Oktober 2014}

Messe Zürich, 9.00 bis $17.00 \mathrm{Uhr}$

Mit ca. 370 Ausstellern rund um die Schwerpunktthemen Medizintechnik, Diagnostik, Rehabilitation, Pflege, Verbrauch, Informatik, Organisation, Einrichtung, Dienstleistungen und Verlagserzeugnisse. nicht gefährdet. Gemeinschaftspraxen bieten bei seriellen Arbeitszeitmodellen längere Öffnungszeiten. Der grösste Vorteil liegt laut Schnidrig aber in der Möglichkeit der integrierten Versorgung. Patienten finden in Praxisgemeinschaften aus Allgemein-, Fach- und Paramedizinern, zum Beispiel Physiotherapeuten, medizinische Dienstleistungen aus einer Hand. Schnidrig betont: «Gesundheitszentren bieten den Vorteil, dass Diagnosetests nur noch einmal, nämlich vom behandelnden Allgemeinmediziner durchgeführt werden.» Deshalb setze man ganz bewusst auf eine integrierte Versorgung von Patientinnen und Patienten.

\section{Mehr Zeit, höhere Effizienz und tiefere Kosten}

Die Vorteile von Gemeinschaftspraxen liegen auf der Hand: Ärztinnen und Ärzte haben mehr Zeit für ihre Patientinnen und Patienten, weil die Administration zentral von internen oder externen Dienstleistern geführt wird. Sie müssen sich nicht mehr verschulden und können wertvolle Erfahrungen sammeln. Auch selbständige Ärzte, die gemeinsam eine Praxis oder ein Gesundheitszentrum führen, profitieren von Synergieeffekten. Die Nutzung von gemeinsamen Laboreinrichtungen und anderen medizinischen Apparaturen wie auch einer gemeinsamen, allenfalls ausgelagerten IT spart Kosten und Nerven.

Die Digitalisierung in der Medizin schreitet unaufhaltsam voran. Umso wichtiger ist es, dass sich nicht jeder Arzt einzeln um diese Themen kümmern muss, sondern vom Know-how interner oder externer Fachleute sowie der Arbeitsteilung mit den Kollegen profitieren kann. Roland Willi wie auch Peter Sauter sehen aufgrund der strukturellen Vorteile von Gruppenpraxen viel höhere Erfolgschancen in Bezug auf die Stellenbesetzung, indem sich Ärztinnen und Ärzte wieder auf ihre Kernkompetenzen fokussieren können: «Bis vor etwa fünf Jahren war die Auslagerung von ökonomischen und administrativen Aufgaben eine Seltenheit», stellt Peter Sauter von «eastcare» fest. Im Zuge des anhaltenden Strukturwandels brauche es eine Professionalisierung, insbesondere im ökonomisch-administrativen Bereich, um effizient und sinnvoll zusammenarbeiten $\mathrm{zu}$ können, so Sauter.

\section{Referenzen}

1 FMH Ärztestatistik 2013. www.ifas-messe.ch
2 Streit S. Moderne Praxisformen. Resultate einer nen und Hausärzten. PrimaryCare. 2011;11:(19):342-3

3 Hostettler S, Kraft E. Neuste Zahlen und Übersicht zur Ärztestatistik 2013. Schweiz Ärztezeitung. 2014;95(12): 467-73.

4 Hostettler S, Kraft E. Ärzteschaft in der Schweiz - die Feminisierung der Medizin. Schweiz Ärztezeitung. 2009;90(47):1823-5. schweizweiten Umfrage unter zukünftigen Hausärztin- 